\title{
CONGENITAL ANTERIOR ANGULATION OF THE TIBIA
}

\author{
BY \\ J. P. BOUND, H. V. L. FINLAY and F. CLIFFORD ROSE \\ From the Paediatric Department, Hillingdon Hospital, Middlesex
}

(RECEIVED FOR PUBLICATION SEPTEMBER 25, 1951)

It is proposed in this paper to present two cases of congenital anterior angulation of the tibia. Surprisingly little mention is made in standard paediatric textbooks of this condition, which is probably more common than is generally believed. Further, speculation as to its cause may be of value at the present time when antenatal paediatrics is- arousing such widespread interest and when prevention of certain congenital deformities is becoming more than a mere possibility.

The vast majority of cases of congenital anterior angulation of the tibia reported in the literature occurred in association with a congenital absence of the fibula.

The earliest reference to congenital anterior angulation of the tibia which it has been possible to trace is a report by Proudfoot in 1846 of a - case of compound fracture of the tibia in utero and congenital talipes talus:. In all probability there was an associated fibular maldevelopment in this case. On reading the first two paragraphs of Proudfoot's article, which are worthy of quotation in full, one is not surprised that the erroneous diagnosis of intra-uterine fracture was made.

\footnotetext{
- Mrs. F., age 28 years, of sanguine temperament, and who has always enjoyed good health, during the sixth month of gestation, and of her third pregnancy, while attempting to pass through a very narrow passage, was severely pressed on the abdomen, and immediately experienced a violent pain in that region, accompanied with nausea and faintness. The following day she was threatened with abortion, having pain and some haemorrhage. These symptoms continued for some days, and occasionally reappeared in a light degree, up to the time of her delivery, which occurred at full time and was perfectly natural. At birth, the right foot of the child (a female) was found permanently extended and turned upward, pressing against the side of the leg, above the external malleolus.

The tibia of the same side, about the middle of the bone, had apparently been the seat of a compound fracture; the two ends had united, at a slight angle anteriorly, and opposite to this angle there was a cicatrix, evidently of some time standing; there was
}

considerable thickening of the parts near the seat of injury.'

In 1898 Cotton and Chute had gathered together and analysed no fewer than 128 cases of congenital absence of the fibula. The number of published case reports appearing thereafter in the literature may be gauged from the fact that Lapasset and Cahuzac in 1935 had found records of 295 cases of congenital absence of the fibula.

Kirmisson (1898) gave a full clinical description of congenital absence of the fibula in his textbook, adding six cases of his own to the literature, which he reviewed. Complete absence of the fibula was more common than partial absence and the condition was more often unilateral than bilateral. Anterior angulation of the tibia at the junction of its upper two-thirds and lower third was a usual association. The summit of the angle commonly pointed inwards and this, together with retraction of the tendo Achillis, resulted in an equino-valgus position of the foot. Overlying the angle, a "scar' was constantly found. Absence of one or more toes with the corresponding metatarsals was common, usually those of the lateral side of the foot. The whole limb was atrophied to some extent. Other malformations of the affected limb were not rare; shortening of the thigh, dislocation of the hip and genu valgum. Congenital deformities elsewhere in the body had also been associated, such as cleft palate and complete or partial absence of the radius.

Discussing the pathogenesis, Kirmisson argued convincingly against the condition being caused by intra-uterine fractures, under which heading these cases had previously been described. He thought the most tenable theory was that which supposed a mechanical cause operating during the first two months of pregnancy, the result of pressure exerted by a small amniotic sac, often combined with the effect of amniotic adhesions. He thought these adhesions were also responsible for the scars. 
Kirmisson also noted the existence of cases of angulation of the tibia but with an otherwise normal skeleton. In some of these, intra-uterine fracture was a possibility as a pseudarthrosis was present at the point of angulation, but he did not think this a reason for rejecting the pressure theory. The prognosis depended on the amount of atrophy of the limb.

Potel (1925) gave a more detailed description of cases in which both tibia and fibula were present. He did not believe the theory of amniotic compression, but thought the deformities were due to a lesion of the mesoderm during its differentiation. The bending was attributed to the lengthening bone being restrained by a lesser development of the surrounding muscle mass. The scar was simply due to stretching of the skin, or perhaps to amniotic adhesions in some cases.

In his studies on pre-natal lesions of striated muscle as a cause of congenital deformity, Middleton (1932, 1934) published three further cases of congenital angulation of the tibia, one of which was associated with radiological absence of the fibula. He preferred the term 'congenital tibial kyphosis: In an attempt to explain the associated talipes equinus which was apparently produced and maintained by the short calf muscles, Middleton relegated the bony angulation to a secondary place and viewed the muscular shortening as the primary factor. He supposed that there was a relative failure during intra-uterine life of the last stage of growth (i.e. growth in length) of the developing myocyte, leading to a progressive retraction of the tendo calcaneus, and hence to the production of an equinus deformity at the ankle joint. When the ankle joint had become plantarflexed to its fullest extent, the process of relative shortening continuing, the strain fell upon the young shaft of the tibia, which being still cartilaginous, naturally bent at its weakest point which is the junction of the middle and distal thirds. With the advent of full ossification of the diaphysis of the tibia the process of progressive deformity came to an end. The dimples were said to mark the spots where bursae were likely to develop in later life.

Browne (1936) put forward strong arguments in favour of a mechanical origin for many congenital deformities, including "fracture of the tibia". He suggested that this was due to the effects of normal mechanical and hydraulic pressure in association with faulty development of the bones, the weaker leg being bent over the other. He thought that skin dimples also were the result of pressure and occurred over bony points in contact with the uterine wall.

Freund (1936) pointed out that most of the reported cases of congenital absence of the fibula occurred in young children who may show total absence of the fibula on clinical and radiological exanination, but a few years later present a rudimentary fibula in the radiograph. He believed that the diagnosis of congenital absence of the fibula should not be made before the fifth year of life at the earliest.

Chapple and Davidson (1941) had their interest aroused in the subject of the relationship between foetal position and certain congenital deformities by a male infant who was born with marked lateral bowing of both femora, laterally dislocated hips, shallow acetabula, but a skeleton otherwise normal. When the infant was folded so that each foot opposed the apex of the lateral curvature of the other thigh, drawing the legs up tailor-fashion, with each leg meeting the opposite femur at a right angle, it was found that the baby preferred this position which they termed 'the position of comfort'. They stressed the importance of determining the position of comfort as a routine part of the examination of newborn infants, both as a means of anticipation and prevention of bone and joint abnormalities and as an aid to the interpretation of existing deformities.

Williams (1943) added a further two cases of congenital angulation of the tibia to the literature, the first patient survived only $\mathbf{3 0}$ minutes and showed normal fibulae. The second revealed radiological absence of the fibulae and, after amputation had proved necessary, the distal portions of the limbs were dissected. There was a solid, strong fibular shaft of fibro-cartilage with absence of ossification and great predominance of the fibrous element. Histological examination of one of the skin dimples showed that only the elastic tissue was slightly deficient and that the deep layers of the true dermis were in immediate apposition to the periosteum over the bony cortex. Williams still adhered to the theory of a basic mesenchymal defect. This resulted in a limitation of muscle growth, producing the angulation, and a failure of transitional differentiation to fibrocartilage, cartilage and true bone, causing the frequently associated defects. He stated that treatment was difficult and the functional outlook poor.

In a paper entitled 'Prenatal Bowing and Thickening of Tubular Bones with Multiple Cutaneous Dimples in Arms and Legs', Caffey (1947) published three cases in which there was symmetrical lateral bowing of the femora and symmetrical posterior bowing of the humeri. Distal to the knees and elbows there were similar, but usually unpaired, deformities of the tibiae, 
radii and ulnae. The fibulae were present in all his cases. Large symmetrically-placed dimples overlay the salient angle in several of the deformed bones of his first two cases. At the level of the bone angulation there was shown radiologically a combination of cortical thickening on the concavity with cortical thinning on the convexity, with reduction in the calibre of the medullary cavity.

Caffey explained the cortical hypertrophy as being due to failure of absorption from within, and not as representing subperiosteal accretion on the external surface. Clinically there was a gradual post-natal regression of the bowing of the bones, especially in the upper limbs, and the cortical thickening tended to lessen. Two of Caffey's cases showed metaphyseal spurs on the medial aspects of the opposing bones at the knees, and he emphasized the similarity to Blount's disease. He particularly drew attention to the occurrence of co-existing symmetrical bowing of the bones of the arms in these cases.

Caffey produced some convincing evidence that the skeletal deformities could be explained satisfactorily in large part by assuming that there had been mechanical pressure operating on ectopic foetal extremities. The deviation from the normal foetal position needed to be only slight. He imagined the foetus packed in a cross-leg and crossarm position, in which each foot pressed on the internal aspect of the opposite thigh, and each hand on the flexor surface of the opposite upper arm. The bone bowed at the site of impact and the skin caught between the summit of the curve and the uterine wall, underwent pressure atrophy which produced a dimple. Caffey considered that Middleton's hypothesis did not fit his cases as there was no evidence of muscle shortening, though he did not rule out the possibility that transient muscle shortening of early foetal life could produce persistent curvature of bone.

Recently Duraiswami (1950) has induced various skeletal abnormalities in the developing chick embryo by the use of insulin. One of his radiographs of a 3-week-old chicken shows 'pathological fractures ' of both tibiae with marked buttressing of the concavities of the bowed bones by subperiosteal new bone, insulin having been injected into the egg on the fourth day. Duraiswami was of the opinion that these skeletal deformities were due to an unchecked insulin hypoglycaemia acting on the embryonic cells during the early days of incubation.

\section{Case Reports}

Case 1. R.J., a full-term girl, weighing $8 \mathrm{lb} .14 \mathrm{oz}$, was born in Hillingdon Hospital on September 14, 1950. The mother was 39 years old and had had three normal children. There was no history of maternal illness during the first three months of pregnancy. At the 29th week of pregnancy a radiograph of the mother's abdomen was taken because it was thought to be unduly large. and twins were suspected: this revealed bilateral angulation of the humeri, femora, tibiae and fibulae of the foetus. In this projection no angulation of the radii and ulnae could be seen. Delivery was normal but the infant was asphyxiated and died 15 hours later.

The limbs showed bilateral talipes equinus and anterior bowing of the lower legs. There was a cutaneous dimple over the site of angulation on the anterior surface of each leg. Palpation revealed that the dimple overlay a bony prominence on the anterior border of the tibia. Similar dimples overlying bony prominences were present on each forearm.

Radiography (Dr. S. A. Maddocks) showed slight cortical thickening posteromedially in the femora and angulation of each tibia at the junction of its upper twothirds and lower third, while the point of fibular angulation was slightly more proximal. Both tibiae and fibulae showed posterior cortical thickening. Each arm (Fig. 1) showed bony projections on the radius and ulna at the site of a mild angulation, with lateral cortical thickening on the right side. In this projection no angulation of the humerus

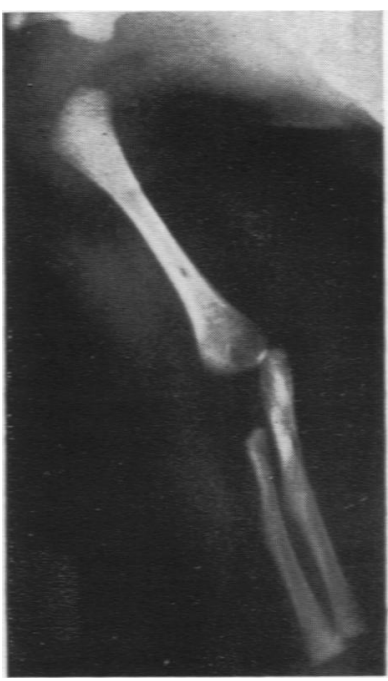

FKG. 1.-Radiograph (Case 1) of right arm, showing bony projections on the radius and ulna at the site of a mild angulation. was evident.

A post-mortem examination was carried out by Dr. W. H. A. Picton. One tibia and fibula (Fig. 2) were removed, and a radiograph of the specimen clearly demonstrated the bony architecture, with thinning of the cortex on the convex surface and thickening on the concave side (Fig. 3). The necropsy produced evidence that the baby died of asphyxia: both lungs were atelectatic and the brain showed subarachnoid haemorrhages and an area of small haemorrhages in the posterior part of the left internal capsule.

Case 2. M.W., a boy, was first seen at Hillingdon Hospital at the age of 5 months on January 8, 1951, when he was admitted because of collapse of the right upper lobe of the lung. The mother was a primipara aged 28 years. During the pregnancy she had no illness but her friends had remarked on the smallness of her abdomen and she had been able to wear and button up her ordinary coat until term. A normal 
delivery occurred at full term, the birth weight being $6 \mathrm{lb} .7 \mathrm{oz}$.

When the infant was examined several deformities were evident. Both legs were bowed anteriorly and slightly laterally, more so on the right side, and the feet were in a mild equinus position, dorsiflexion being

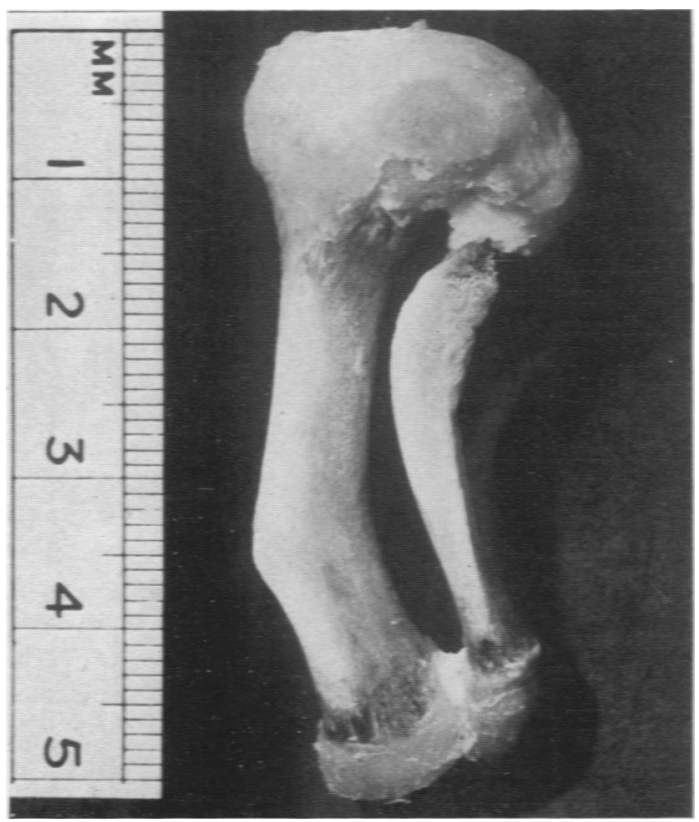

Fig. 2.-Post-mortem specimen (Case 1) showing tibia and fibula.

Fig. 3.-Radiograph (Case 1) of tibia and fibula after post-mortem dissection, showing angulation and bony architecture.

limited to $90^{\circ}$. Each leg showed a pair of cutaneous dimples lying anteriorly at approximately the same level just below the midpoint of the shin, and much deeper on the right side. Beneath the two dimples on each leg were prominences of the anterior borders of the tibia and fibula respectively. A shallow dimple overlay the head of the fifth metatarsal on each side.

The dimples over the external epicondyles of the humeri and the olecranon processes were more marked than normal. The medial three fingers of both hands were fixed in flexion at the metacarpo-phalangeal and proximal interphalangeal joints, the little finger being the most severely affected. The chest showed a marked pigeon-breast deformity and bilateral triangular grooves into which the elbows and forearms fitted when pressed to the sides. The skull was plagiocephalic, the forehe a d be ing prominent on the right side. Micrognathia was a marked feature and there was a cleft of the soft palate.

Radiography showed very slight lateral bowing of the femora, and the point of maximal bending of the tibia on both sides was just below the mid-point of the

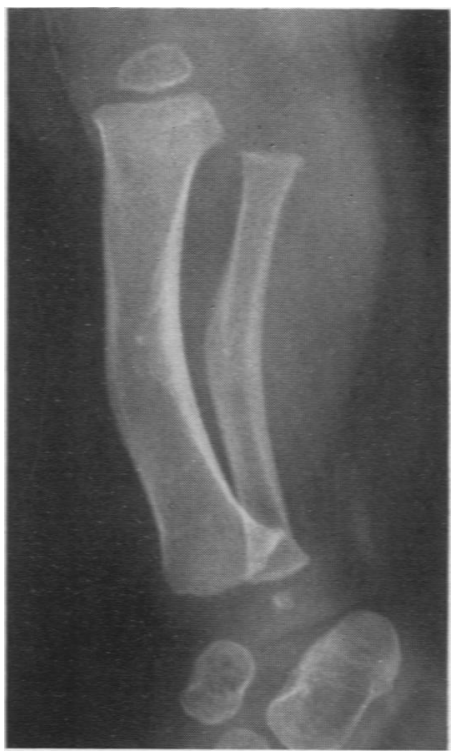

Fig. 4.-Radiograph (Case 2) of right tibia and fibula at the age of 1 year, showing anterior angulation.

bone, and was associated with cortical thickening posteriorly: the anterior prominence was more marked in the fibula than in the tibia (Fig. 4). The left humerus showed mild posterior bowing in its lower half in a lateral view. The skull and spine were normal.

Attempts were made to reconstruct the foetal position of the legs. It was found that they could be folded up easily over the lower abdomen, so that each foot rested against the opposite thigh, without causing any distress to the infant (Fig. 5).

Manipulation and splinting of the fingers have shown an excellent result. After strapping the feet in the early months under the care of Mr. Eyre-Brook, regular manipulatory moulding of the lower legs and feet has been carried out for eight months with a slight improvement in the faulty alignment.

\section{Discussion}

It will be seen from the foregoing accounts in the literature that the condition of congenital angulation of the tibia with ossified fibulae is merely a forme fruste of the more severe and crippling deformity usually produced by congenital absence of the fibula. It has been shown that radiological absence of the fibula may merely indicate delay in ossification of the bone which is still present in cartilaginous or fibrous form.

Before considering some of the many theories put forward to explain the deformities found in this condition, Potel's (1925) words may be quoted: - Que le péroné existe ou non, la coudure tibiale ne saurait avoir deux mécanismes différents et ces 
affections sont absolument superposables et peuvent s'expliquer par un mécanisme identique .

Although clinically angulation of the tibia may be the presenting feature, it must also be remembered that it may be only one manifestation of a pathology

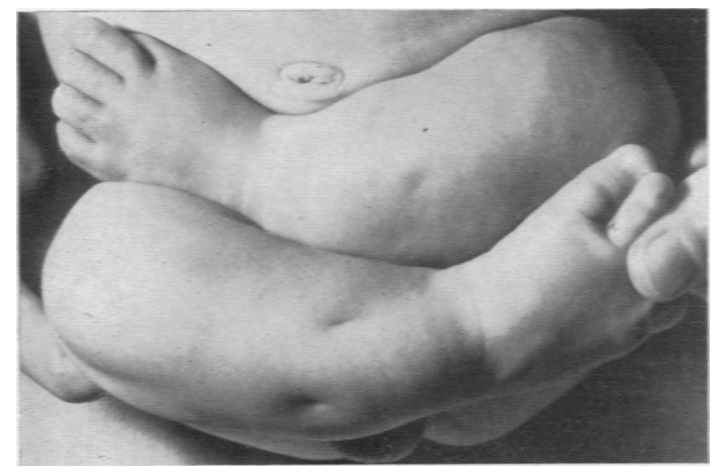

FiG. 5.-Case 2 at the age of 5 months, showing attempt at reconstruction of foetal position.

affecting to a varying degree the long bones of both arms and legs. This has been emphasized by Caffey and is well shown by our first case. Any theory as to aetiology must take this fact into consideration.

The following theories must be considered.

Lnion of an Intra-uterine Fracture. The frequent occurrence of the angulation of the tibia as a bilateral and almost symmetrical deformity by itself renders this theory unlikely, as does the frequent presence of other associated defects. Examination of the bones at the site of angulation does not reveal any sign of a pre-existing fracture either radiologically or pathologically, although this does not preclude the possibility of fracture having occurred very early in foetal life.

Heredity. This does not appear to be an important factor. In only a few cases has a familial tendency been recorded: in most the condition appears unexpectedly in otherwise healthy stock.

Intra-uterine Failure of Growth in Length of Developing Muscle Unit. This theory as put forward by Middleton $(1932,1934)$ has already been described. One of the objections to it is that cases have been described without talipes, while in our second case only a mild talipes was present: if the shortening of the calf muscles was sufficient to cause bowing of the tibia, an extreme degree of talipes equinus would be expected. The theory offers no satisfactory explanation for the presence of the dimples.

Excessive Intra-uterine Pressure. This conception is not modern but fell into disrepute until recent years when attention was again drawn to it. That excessive intra-uterine packing can cause foetal deformities is an attractive theory which can explain most of the facts. Ideas as to the precise mechanism of the process are vague, but it seems not unreasonable to suppose that in certain cases the growth of the surrounding tissues fails to keep pace with that of the foetus in the amniotic cavity. If this difference in growth is considerable and occurs in the early weeks of pregnancy while the foetal bones are still cartilaginous, impairment of the vascular supply to the lateral side of the legs and feet, with consequent defect of fibular growth and absence of the lateral toes, might result. At the same stage, lesser degrees of packing might produce bending of the long bones only. On the other hand, pressure of any degree occurring somewhat later in pregnancy could produce bending of already ossifying long bones. Such 'pressure' occurring early in pregnancy may be only transient. As Gaenslen (1915) has said, the original deficiency of amniotic fluid may be more than made up later in pregnancy. Our first case, if a result of pressure, would be an example of this. The mother's abdomen was thought to be unduly large when she came under observation, but at that time the deformities had already developed. Thus the absence of a small abdomen in the later months does not necessarily exclude mechanical pressure as a cause of foetal deformities. The size of a mother's abdomen throughout pregnancy, however, depends on so many factors that one should hesitate to ascribe too much significance to this observation in relation to foetal deformities.

The multiple deformities of our second case could be readily explained on the theory that increased pressure was exerted on the foetus from the early weeks onwards. Thus the tibial bowing would be due to pressure exerted through the long axis of the bone, the knee being in contact with the uterine wall and the foot pressed against the opposite thigh. The association of micrognathia and cleft palate has been attributed to acute flexion of the head on the chest which results in underdevelopment of the mandible: the mouth does not provide ample space for the tongue and fusion of the palate is prevented (Chapple, 1950). Finally, stiff joints have been thought to be due to interference with the circulation by increased pressure (Browne, 1936): the fingers in our case would be flexed and pressed against the thorax.

Biochemical or Hormonal Influences. Reference has been made to the studies on the chick embryo by Duraiswami (1950). The production of 
deformities of the tibia in some of the insulintreated group is of great interest but it remains to be seen to what extent these findings apply to human embryology.

In order to throw more light on the aetiology of this condition, information about the conditions of pregnancy in further cases is needed. As suggested by Browne (1951), this study should include the mother's sensations during pregnancy, the size of her abdomen and the amount of amniotic fluid. In the baby, the distribution of deformities and dimples, and an attempt to determine the position of comfort are important. Enquiry should also be made about illnesses in early pregnancy which might come to have aetiological importance in relation to a lesion such as that envisaged by Middleton.

\section{Summary}

Two cases of congenital bilateral anterior angulation of the tibia with fully ossified fibulae are described. In the first case angulation of other long bones was present.

The more important papers in the literature are reviewed, most cases being described in association with congenital absence of the fibula.
Cases with congenital bowing or even angulation of the bones of the arms and legs may have tibial angulation as the only obvious clinical feature. The aetiology of the condition is discussed.

We wish to thank Dr. W. Arklay Steel for his advice in the management of the second patient, and we are grateful to Professor J. L. Henderson and Dr. C. G. Barnes for their many helpful suggestions.

The photographs are the work of Mr. E. Stride.

\section{ReFERENCES}

Browne, D. (1936). Proc. roy. Soc. Med., 29, 1409. (1951). Brit. med. J., 1, 247.

Caffey, J. (1947). Amer. J. Dis. Child., 74, 543.

Chapple, C. C. (1950). In Mitchell-Nelson 'Textbook of Pediatrics,' 5th ed., p. $1469 . \quad$ Philadelphia.

and Davidson, D. T. (1941). J. Pediat., 18, 483.

Cotton, T. J. and Chute, A. L. (1898). Trans. Amer. orthop. Ass., 11, 316.

Duraiswami, P. K. (1950). Brit. med. J., 2, 384.

Freund, E. (1936). Arch. Surg., Chicago, 33, 349.

Gaenslen, F. J. (1915). Amer. J. orthop. Surg., 12, 453.

Kirmisson, E. (1898). 'Traité des Maladies Chirurgicales d'origine congénitale,' pp. 551-573. Paris.

Lapasset, and Cahuzac, (1935). Rev. Orthop., 22, 110.

Middleton, D. S. (1932). Edinb. med. J., 39, 389.

(1934). Ibid., 41, 401.

Potel, G. (1925). 'Traité Pratique d'Orthopédie,' pp. 74-81. Paris.

Proudfoot, L. (1846). N. Y. St. J. Med., 7, 199.

Williams, E. R. (1943). Brit. J. Radiol., 16, 371. 